\title{
Improving Infant and Maternal Health through CenteringPregnancy: A Comparison of Maternal Health Indicators and Infant Outcomes between Women Receiving Group versus Traditional Prenatal Care
}

\author{
Ruth Zielinski1,2, Leslie Stork3 ${ }^{3}$, Megan Deibel1,2, Catherine L. Kothari', Kimberly Searing3 \\ ${ }^{1}$ School of Nursing, University of Michigan, Ann Arbor, USA \\ ${ }^{2}$ Borgess Women's Health, Kalamazoo, USA \\ ${ }^{3}$ Bronson School of Nursing, Western Michigan University, Kalamazoo, USA \\ ${ }^{4}$ School of Medicine, Western Michigan University, Kalamazoo, USA \\ Email: ruthcnm@umich.edu
}

Received 17 April 2014; revised 15 May 2014; accepted 23 May 2014

Copyright (C) 2014 by authors and Scientific Research Publishing Inc.

This work is licensed under the Creative Commons Attribution International License (CC BY). http://creativecommons.org/licenses/by/4.0/

\section{Open Access}

\section{Abstract}

Background: Despite efforts to increase participation in prenatal care, outcomes for women and infants in the United States remain below global and national health targets. CenteringPregnancy, a model of group prenatal care, incorporates practices consistent with national and international guidelines while allowing for greater freedom in providing content tailored to the specific needs of women receiving care. Objective: To determine whether the CenteringPregnancy model improves maternal and neonatal health indicators such as prenatal care attendance, smoking cessation, weight gain during pregnancy, gestational age at delivery, mode of delivery, and initiation and continuation of breastfeeding. Methods: A retrospective study was conducted including all pregnant women participating in CenteringPregnancy at two prenatal clinic sites in southwest Michigan from January 2010 to April $2012(n=173)$. A comparison group of women receiving traditional care from certified nurse-midwives was created using propensity scores to match for age, race, and insurance status $(n=170)$. A chart review was performed to analyze maternal and neonatal health indicators including attendance at prenatal visits, gestational age at delivery, baseline maternal weight and weight gain during pregnancy, smoking cessation, infant birth weight, mode of delivery (vaginal birth vs. cesarean section), and rates of breastfeeding. Results: There were no significant differences in pre-pregnancy weight, amount of weight gained during pregnancy, prenatal care attendance, gestational age at delivery, mode of delivery or infant birth weight. The 
CenteringPregnancy group had significantly higher rates of smoking cessation during pregnancy, as well as higher rates of breastfeeding initiation and continuation. Conclusions: This study provides support for the benefits of CenteringPregnancy in improving rates of smoking cessation during pregnancy which is important to both maternal and infant health. Additionally, in this population CenteringPregnancy resulted in improved rates of breastfeeding initiation and continuation, providing benefits to both infants and mothers.

\section{Keywords}

CenteringPregnancy, Prenatal Care, Breastfeeding, Group Care, Smoking Cessation, Pregnancy

\section{Introduction}

By many measures the United States (US) continues to have less than optimal outcomes related to pregnancy and birth. The US ranks an estimated $46^{\text {th }}$ in infant mortality and $6^{\text {th }}$ in maternal mortality rates when compared with other countries [1]. While there has been a recent drop in prematurity rates, the US continues to lag behind all of the Western European countries when it comes to infants born prematurely or with low birth weight [2]. These poor outcomes persist despite spending more healthcare dollars per birth than any other country [3]. Racial and ethnic disparities in society and healthcare contribute to even greater rates of preterm births and other adverse perinatal outcomes within certain populations [4].

Conventional wisdom held that prenatal care, widely implemented since the 1980s in the US, would be beneficial in improving maternal and neonatal outcomes. The traditional model of prenatal care utilized by most providers in the US has changed little since the 1930s. This model involves monthly visits until 28 weeks gestation, followed by appointments every two weeks until 36 weeks gestation, at which point the recommendation is one visit per week until delivery [5]. Typically these visits are short, lasting about 10 minutes and focus on risk assessment. Usually there is little education provided to pregnant women about health-promoting behaviors [6]. The effectiveness of this standardized approach of prescribed numbers of visits at prescribed times in the pregnancy has been called to question for "operat(ing) under the assumption that all women will benefit from routine, ritualized medical encounters" ([5] p. 279). Prenatal care is intended to be a valuable form of preventative healthcare that reduces the risks of eclampsia, low birthweight, prematurity, and infant mortality, while educating the mother about appropriate health-promoting behaviors. However most research on prenatal care has focused on attempting to establish a causal and dose-dependent relationship between the number of visits and the health outcomes, leaving out a very important consideration; the actual content of care is provided in those visits [7].

CenteringPregnancy is an innovative model of prenatal care involving group visits that maintain the risk assessment components of prenatal care while providing greater opportunities for education and support during prenatal care visits [8]. CenteringPregnancy combines health assessment, education and support through a stable group composition and facilitated discussions (versus lecturing). Cohorts of 8 - 12 women are formed, all of whom have similar estimated dates of delivery. These women meet monthly with their provider from approximately 12 - 14 weeks gestation until around 35 weeks, and about every two weeks after that [8]. In 2012, the Institute of Medicine (IOM) issued guidelines for patient-centered care including allowing patients the opportunity to fully participate in their care and health decision-making [9]. The CenteringPregnancy model closely follows the IOM guidelines for prenatal care, including use of continuous, healing relationships and customization of care based on the patient's needs and values.

Since its inception, studies of CenteringPregnancy indicate that this model of prenatal care may result in improved outcomes for both mothers and infants. These benefits include: improved attendance at prenatal visits [10]-[13], increased maternal weight gain [11] [12], lower rates of preterm births [10] [13], increased satisfaction with prenatal care [10] [11] [13], greater maternal knowledge of pregnancy [14] [15], increased rates of vaginal delivery [12], decreased reports of postpartum depression [16], and higher rates of breastfeeding initiation [11] [17]. In some studies, participation in CenteringPregnancy resulted in increased maternal weight gain [11] [12] although whether or not more women in CenteringPregnancy achieved optimal weight gain based on prepregnancy BMI was not identified. Additionally, the CenteringPregnancy model of group care has been shown 
to be beneficial in preparing families for birth [18].

In 2009 CenteringPregnancy was implemented in a certified nurse-midwifery (CNM), hospital-based practice located in southwest Michigan. Since implementation, pregnant women choosing CNM care are offered CenteringPregnancy at their initial intake visit conducted with a nurse. Participation in CenteringPregnancy is voluntary and is offered to all women who are no more than 20 weeks gestation and eligible for CNM (vs physician) care. The population in this community is estimated to be $82.8 \%$ Caucasian, $11.1 \%$ African American, $2.4 \%$ Asian, and 4.1\% Hispanic [19]. At 7.2 per 1000 live births, Michigan's infant mortality rate is higher than the national average of 6.15 per 1000 [20]. Furthermore, in the county where most of the participants in this study reside, the infant mortality rate is even higher at 7.7 per 1000 [21]. The population in this county is at risk for undesirable pregnancy, birth, and infant health outcomes due to a high rate of poverty (18.6\%), which is above both the national (15.0\%) and state (15.7\%) averages [22]. In the state of Michigan, approximately $27 \%$ of pregnant mothers smoke at the time of pregnancy diagnosis, with $11 \%$ quitting smoking when they discover they are pregnant and 15.9\% still smoking during the third trimester [23]. The Michigan Department of Community Health conducted a survey in 2008 and found that the rate of breastfeeding initiation was 73.4\% [24], falling short of the Healthy People 2020 goal of 81.9\% [25]. Exclusive breastfeeding at three months of age was only 31.0\% in the state of Michigan [24] while the Healthy People 2020 goal for exclusive breastfeeding at three months is $42.2 \%$ [25].

The suboptimal outcomes within this community indicate a need for intervention. An alternative model of prenatal care such as CenteringPregnancy has the potential to improve outcomes for women and infants. The purpose of this study was to explore the influence of CenteringPregnancy in this population of women and to identify whether the benefits found in prior studies will translate to this population. Additionally, we were hoping to see if additional benefits such as smoking cessation would be identified in this population. Specifically, we compared select pregnancy outcomes between women receiving traditional prenatal care and women participating in CenteringPregnancy in this nurse-midwifery practice to see if CenteringPregnancy could improve these outcomes. Variables explored in this study include: adequacy of prenatal care, weight gain during pregnancy, smoking cessation, gestation at delivery and breastfeeding initiation and continuation to six weeks.

\section{Methods}

\subsection{Design and Data Collection}

This study was a case-control retrospective study of CenteringPregnancy participants and a comparison group of women receiving traditional CNM care, matched on age, insurance and race using propensity score matching. Study data was generated through retrospective chart review of prenatal and intra-partum visit records. These records included initial prenatal visit with obstetric and health history as well as follow up visits, delivery records and post partum office visits. Structured abstraction forms were developed, piloted and revised by four of the five study investigators through an iterative, consensus-based process. Once finalized, three of the investigators completed the record abstraction. Institutional Review Board approval was obtained from both the university as well as the hospital where care was obtained. As this was a retrospective chart review with data entry and analysis conducted with de-identified data the requirement for informed consent was waived by the Institutional Review Boards.

\subsection{Participants}

Study participants delivered at the same hospital and received their prenatal care from one of two sites where both CenteringPregnancy and traditional care with CNMs was offered. During the study time period (from January 1, 2010 to April 30, 2012) 173 women received their prenatal care through CenteringPregnancy and delivered infants (the intervention group). Inclusion criteria for the comparison group were delivery at the same hospital and receiving traditional care with CNMs. During the same time period a total of 1427 women received traditional prenatal care from CNMs and delivered an infant at the same hospital. To produce a matched control group, logistic regression was conducted on the entire delivery population that produced a propensity score representing the likelihood of being a CenteringPregnancy participant based upon the matching criteria (e.g., age, insurance, race). Then, for each CenteringPregnancy participant, a traditional-care participant was selected who had the closest propensity score (i.e., was the closest match on age, insurance and race). It was not possible to 
conduct a randomized, controlled trial because women receiving care in this CNM practice self-select into either CenteringPregnancy or traditional care. Therefore, we utilized a matched comparison group $(n=170)$ using propensity scores in order to reduce the effects of age, insurance type (as a proxy for income) and race on the outcomes we wished to measure [26]. Group sizes were slightly dissimilar because three in the traditional care group were found to have not met all criteria on further analysis (not CNM care or delivering at the study hospital).

\subsection{Variables and Statistical Analysis}

Prenatal data collected included; pre-pregnancy weight, weight gain during pregnancy, gestational age at first prenatal visit, and self-report of ever smoking during this pregnancy and smoking cessation (at pregnancy diagnosis and later, during the pregnancy). Intra-partum data collected included; gestation at time of birth and mode of delivery (vaginal vs. cesarean section). We also collected data on breastfeeding initiation in hospital and continuation at the 6-week postpartum visit as well as attendance rates at the 6-week postpartum visit. Data were entered into SPSS version 18 and analysis included descriptive statistics, independent t-tests and Chi Square. All levels of significance were two tailed and set at $\mathrm{p}<0.05$.

\section{Results}

Mean age for the experimental group (CenteringPregnancy) participants was 24.2 years and the comparison group mean age was $25.5(\mathrm{p}=0.497)$. In both groups the percentage of women having private insurance and those with Medicaid were nearly equal (Table 1$)$. The majority of the women were Caucasian $(78.2 \%$ in the comparison group and $76.9 \%$ in CenteringPregnancy). We did not anticipate between-groups differences on these demographics because we intentionally matched on these three variables when forming the comparison group (Table 1). Additionally, there was not a statistically significant difference in number of prior pregnancies with the mean parity of the comparison (traditional care) group being 0.78 prior births and the mean parity for the CenteringPregnancy group being $1.1(\mathrm{p}=0.227)$. There were two women $(1.2 \%)$ in each group who had experienced a prior preterm birth.

There was a significant between-groups difference in the gestation of pregnancy at first prenatal visit (Table 2). The mean gestational age for the CenteringPregnancy group at first visit was 10.3 weeks versus a mean of 11.8 weeks for the traditional care group $(p=0.031)$. CenteringPregnancy participants were not statistically different from traditional care participants regarding number of prenatal visits.

Table 1. Participant demographics and obstetric history.

\begin{tabular}{|c|c|c|c|}
\hline & \multicolumn{3}{|c|}{ Participant Group } \\
\hline & Traditional Care $(\mathrm{n}=170)$ & Centering $(\mathrm{n}=173)$ & $p$ Value \\
\hline Age & Mean $=25.5($ SD 4.36$)$ & Mean $=24.2($ SD 5.3) & $\mathrm{p}=0.497$ \\
\hline \multirow{2}{*}{ Insurance } & Private: $49 \%$ & Private: $49.5 \%$ & \multirow{2}{*}{$\mathrm{p}=0.479$} \\
\hline & Medicaid: 51\% & Medicaid: 50.5\% & \\
\hline \multirow{3}{*}{ Ethnicity } & White: $78.2 \%$ & White: $76.9 \%$ & \multirow{3}{*}{$\mathrm{p}=0.899$} \\
\hline & Black: $17.6 \%$ & Black: 16.7 & \\
\hline & Other: $4.2 \%$ & Other: $6.4 \%$ & \\
\hline
\end{tabular}

Table 2. Prenatal care.

\begin{tabular}{cccc}
\hline & & Participant Group & \\
\cline { 2 - 4 } & Traditional Care $(\mathrm{n}=170)$ & Centering (n = 173) & $p$ Value \\
Gestation at First Visit & $11.82($ SD 8.7$)$ & $10.30($ SD 3.1) & $\mathrm{p}=0.031$ \\
Total Number of PN Visits & $13.4($ SD 10.7) & $14.2($ SD 7.2) & $\mathrm{p}=0.266$ \\
\hline
\end{tabular}


There was not a significant between groups difference in the number of women who self-reported smoking at time of pregnancy diagnosis (26\% of those who chose CenteringPregnancy and $30 \%$ of those who chose traditional care, $\mathrm{p}=0.272$ ). There was, however, a significant difference in the percentage of women who quit smoking during pregnancy with $84 \%$ of participants in the CenteringPregnancy group and $24 \%$ of women receiving traditional care reporting smoking cessation during pregnancy $(\mathrm{p}<0.000)$. The data was further analyzed to identify the proportion of women who quit smoking at pregnancy diagnosis versus those who quit during the course of their prenatal care. More women who subsequently chose CenteringPregnancy quit smoking at pregnancy diagnosis $(69 \%)$ than those who subsequently chose traditional care $(18 \%, \mathrm{p}<0.000)$. However, during the course of prenatal care $50 \%$ of the women who had continued to smoke past pregnancy diagnosis quit in the CenteringPregnancy group versus only $8 \%$ of those receiving traditional care $(\mathrm{p}<0.000$, Table 3 ).

A similar proportion of women in both the CenteringPregnancy group (42\%) and the traditional care group (41\%) were considered of normal pre-pregnancy weight by Body Mass Index (BMI) category (18.5 - 24.9). Four percent of women in both groups met the criteria for underweight (BMI less than 18.5). In the traditional care group 30\% were overweight (BMI between 25 and 29.9) and 25\% were obese (BMI 30 or greater). In the CenteringPregnancy group there were similar percentages with $29 \%$ overweight and $25 \%$ obese (Figure 1). There was no difference in mean weight gained during pregnancy between groups (33.1 lbs. for CenteringPregnancy and $33.7 \mathrm{lbs}$. for traditional care, $\mathrm{p}=0.841$ ). Recent guidelines from the IOM include recommendations for weight gain during pregnancy based on BMI category prior to pregnancy [27] therefore the data was further evaluated based on these recommendations. The recommendations are that women who are underweight gain 28 - $40 \mathrm{lbs}$., normal weight women gain 25 - 35 lbs., women who are overweight gain 15 - 25 lbs. and women who are obese gain 11 - 20 lbs. [27]. Using the IOM guidelines for weight gain during pregnancy, there was no difference in women who gained the recommended amount of weight for their pre-pregnancy BMI category between groups ( $25 \%$ in Centering vs $28 \%$ in traditional care, $\mathrm{p}=0.244$ ). For both groups the largest category was women who gained more than the recommended amount for their BMI category (53\% of the women in CenteringPregnancy and $49 \%$ of the women in traditional care, Figure 2).

To determine if there was a difference in rates of preterm birth, we compared the number of women who delivered prior to 37 weeks in the CenteringPregnancy and in the traditional care group. Ten infants were born prior to 37 weeks in each group, equaling 5.8\% of the total births in the CenteringPregnancy group and 5.9\% in the traditional care group. No significant difference was found in gestational age at time of delivery with a mean gestational age of 39.45 weeks for CenteringPregnancy participants and 39.5 weeks for traditional care participants $(\mathrm{p}=0.736)$. Rate of Cesarean section in the CenteringPregnancy participants was $17 \%$ versus at $14 \%$ rate

\begin{tabular}{|c|c|c|c|}
\hline & \multicolumn{3}{|c|}{ Participant Group } \\
\hline & Traditional Care $(\mathrm{n}=170)$ & Centering $(\mathrm{n}=173)$ & $p$ Value \\
\hline Smoker & 49 (30\%) & $45(26 \%)$ & $p=0.272$ \\
\hline Quit with Pregnancy Diagnosis & $9(18 \%)$ & $31(69 \%)$ & $\mathrm{p}<0.000$ \\
\hline Quit during Prenatal Care & $3(8 \%)$ & $7(50 \%)$ & $\mathrm{p}<0.000$ \\
\hline
\end{tabular}

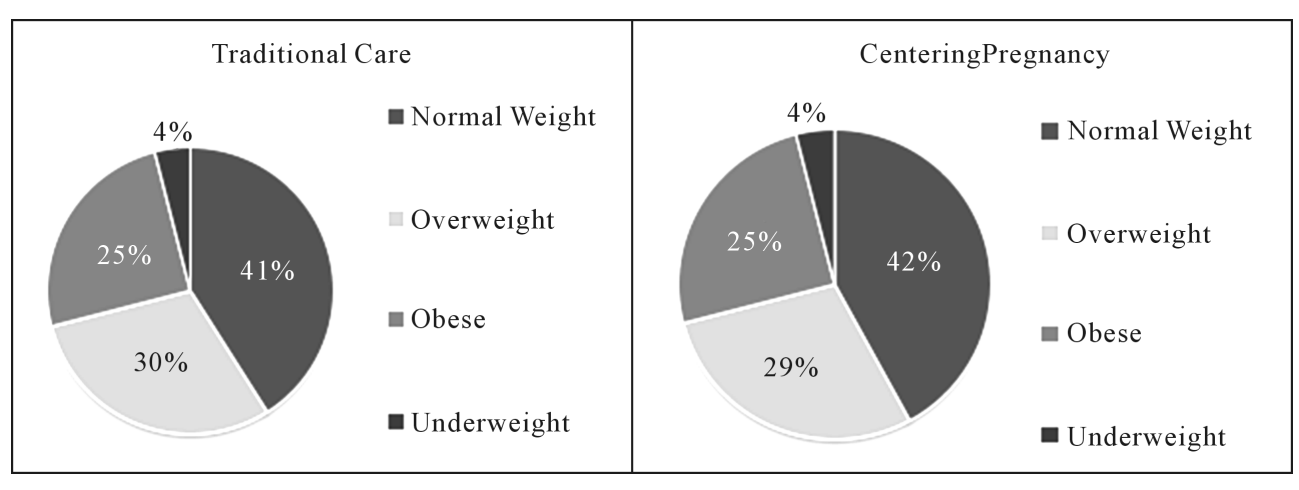

Figure 1. Comparison of pre-pregnancy weight. 


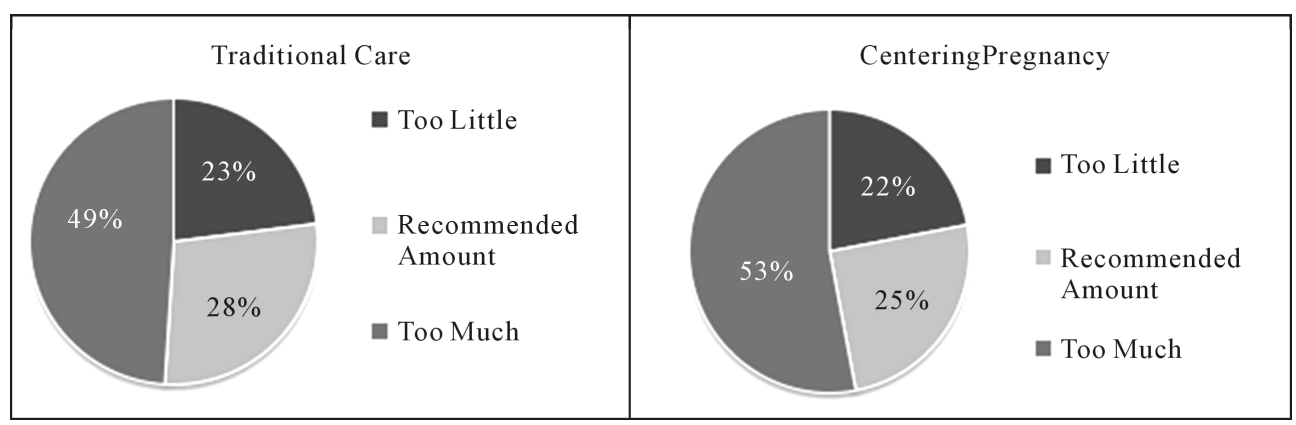

Figure 2. Comparison of weight gain during pregnancy.

in the traditional care, a difference that was not statistically significant $(p=0.443)$. Indications for Cesarean delivery were not analyzed.

Women participating in CenteringPregnancy were more likely to initiate breastfeeding during their hospital stay with an initiation rate of $79 \%$ versus a $60 \%$ rate of initiation in the hospital for the traditional care group (p $=0.001$ ). More than half (54\% of women) in the CenteringPregnancy group were still breastfeeding at 6 weeks postpartum (97/173) versus only $42 \%(72 / 170)$ of the women in the traditional care group $(\mathrm{p}<0.000)$.

\section{Discussion}

Health indicators and behaviors such as maternal smoking, overweight/obesity, pregnancy weight gain and breastfeeding have the potential to influence pregnancy and infant outcomes. The purpose of this study was to determine if CenteringPregnancy resulted in improved health behaviors and outcomes in this population of women and infants.

Cigarette smoking is associated with negative effects such as low birth weight, placental complications and prematurity [28]. Rates of smoking cessation during pregnancy in Michigan fall short of national goals, negatively affecting both the mother's overall health as well as that of her infant [23]. The US Healthy People 2020 initiative [24] included these three goals: reduce the number of women who smoke prior to pregnancy to $14 \%$, increase the number of women who stop smoking during pregnancy to $30 \%$, and reduce the number of women who smoke during pregnancy to $1 \%$. Clearly there needs to be further improvement in smoking cessation rates if we are to meet these goals. There was a significant improvement in the rate of smoking cessation in the CenteringPregnancy group versus the women receiving traditional care. Some of this difference may be attributed to a priori differences in the personal characteristics of women who chose CenteringPregnancy. A larger proportion of this group (versus the traditional care group) stopped smoking before they even began prenatal care. This hints at the possibility of a potential between groups difference that is not controlled by matching on demographic variables as we did. It is possible that women who already possess greater health-related self-efficacy are more likely to both quit smoking and select CenteringPregnancy for their prenatal care. Even so, there were significantly more participants who quit smoking during their prenatal care in the CenteringPregnancy group, indicating that the additional education and social support offered in this model of prenatal care could be beneficial in increasing prenatal health behaviors such as smoking cessation. To our knowledge this is the first study of CenteringPregnancy to find an improvement in smoking cessation during group prenatal care.

Overweight/obesity is also a significant health issue among women of childbearing age. More than a third of women 20 or older in the US are obese, with a similar percentage classified as overweight [29]. Too little weight gain during pregnancy is associated with low birth weight and fetal growth restriction while gaining too much weight during pregnancy is associated with gestational diabetes, large for gestational age infants and subsequent delivery complications [30]. While the benefits of appropriate weight gain in pregnancy are well documented, many women report receiving little or no guidance from healthcare providers about weight gain while pregnant [31]. Prior research has indicated that CenteringPregnancy participation resulted in an increase in weight gained during pregnancy. In this study population there was not a between groups difference in either the amount of weight gain or in percentage of women who gained the optimal amount of weight based on their pre-pregnancy BMI category. Furthermore, a significant number of women in both groups gained more than the recommended amount of weight. Recent evidence suggests that excessive weight gain may result in less than desirable out- 
comes including increased rates of gestational diabetes and cesarean birth [32]. This finding indicates that prenatal care should include greater emphasis on weight gain recommendations and education on nutrition and exercise during pregnancy than what is currently provided.

The US also falls short on national breastfeeding goals. There are numerous benefits associated with breastfeeding, including fewer incidences of ear infections, gastroenteritis, and other infections as well as decreased incidence of Sudden Infant Death Syndrome [33]. Additionally, breastfeeding is associated with a long-term decreased risk of obesity for the infant and improved glucose metabolism and cardiovascular health in the breastfeeding mother [34]. While the most recent data indicates that the majority of women in the US start out breastfeeding their infants (76.9\%), rates of exclusive breastfeeding and rates of breastfeeding at 12 months remain low. Despite an abundance of evidence to support breastfeeding, only $43.0 \%$ of infants are fed any breast milk at six months and breastfeeding rates at one year decrease to $22.4 \%$ [35]. The results of this study indicate that CenteringPregnancy participants had higher rates of breastfeeding. Significantly more women in the Centering Pregnancy group initiated breastfeeding prior to hospital discharge compared with the traditional care group. These findings are similar to prior studies of CenteringPregnancy that have demonstrated improved rates of breastfeeding initiation [17]. Because this study included data from the 6 week postpartum visit, it was also established that there was a significant between-groups difference in the number of women who continued breastfeeding their infants for least six weeks. Prior studies have shown that many women who discontinue breastfeeding often do so in the first few weeks [36], therefore this is an important finding.

In this study women who participated in CenteringPregnancy were earlier in their pregnancy gestation at the time of first prenatal visit. This difference is likely due to the exclusion of women from participating in CenteringPregnancy if they present to prenatal care later than 20 weeks gestation at this CNM practice. Prior research has shown that CenteringPregnancy programs have improved attendance at prenatal visits versus traditional prenatal care. While our results did not find this difference, it may be because both groups of women had relatively high rates of attendance at prenatal visits. CenteringPregnancy requires a larger time commitment for visits (two hours per visit) as well as more structured time and date parameters. These results indicate that these factors do not detract women from attending their prenatal care visits.

In this study there was no difference between groups in gestational age at delivery or the number of preterm births. Prior studies have demonstrated an improvement in preterm birth with participation in CenteringPregnancy. The low rates of preterm birth overall when compared to national rates of preterm birth may be attributed, in part, to the criterion for CNM care in this practice. Women in this practice typically are transferred to physician care if they are considered high risk for preterm birth (multiple gestations, history of multiple preterm births, etc.).

The results of this study must be viewed in light of its limitations. In the care practice where this study was conducted, CenteringPregnancy is optional and offered to all women who present for care with CNMs, eliminating the possibility of randomization. Despite an attempt to control for this by using a matched control group, there may be some differences between groups as a result of this study design. The type of data available for collection was limited by the use of retrospective chart analysis. Rates of smoking cessation were based on women's self-report, and no physiologic measures of nicotine use were included in the study. Therefore it is possible that women in the CenteringPregnancy group felt more pressure to report cessation of smoking in the peer setting versus the individual setting with a care provider only. Additionally, self-report is not always the most reliable source of information. Variables such as patient satisfaction, self-efficacy and social support were not available for collection due to the study design. While the sample size used was sufficient for most analyses, larger samples in future studies could result in significant differences in variables such as preterm birth that were not detected in our study due to the relatively low percentage in both groups. According to policy at this health center, all women who qualify for midwifery care are to be offered the opportunity to participate in CenteringPregnancy. It is not known how consistently this is offered as an option in practice.

\section{Conclusions}

In recent years, the United States has made modest strides in improving pregnancy, birth, and post-natal outcomes for women and babies. Efforts have been made to improve the delivery and content of prenatal care that is provided for women and families. In prior studies, CenteringPregnancy is one model of care delivery that has shown promise toward improved maternal and infant outcomes. This retrospective chart review was undertaken 
with the intention of determining if CenteringPregnancy would be effective in this study site in improving select prenatal and birth outcomes for women and babies.

This study provides additional support for the use of CenteringPregnancy as an alternative method for prenatal care delivery. Our findings suggest that CenteringPregnancy may have a positive effect on health-promoting behaviors, including an increase in smoking cessation and increases in the rates of initiation and continuation of breastfeeding. While these findings are promising, thorough research on CenteringPregnancy is lacking, and larger studies are needed to determine if the effects of CenteringPregnancy found in this study are possible in other settings. Future studies across diverse populations of both women and healthcare providers will be beneficial. Whether CenteringPregnancy results in improved self-efficacy for health related behaviors and improved perceived social support is also of interest. Inclusion of partners and families as participants in CenteringPregnancy research should be considered for future research. As the US moves toward increasing access to care while decreasing healthcare expenses, group approaches such as CenteringPregnancy are a potentially valuable alternative to traditional modes of healthcare delivery.

\section{References}

[1] The World Bank Group (2014) Mortality Rate, Infant (per 1,000 Live Births). http://data.worldbank.org/indicator/SP.DYN.IMRT.IN

[2] WHO (2012) Born Too Soon: The Global Action Report on Preterm Birth. Howson, C.P., Kinney, M.V. and Lawn, J.E., Eds., World Health Organization, Geneva.

[3] Truven Health Analytics Marketscan Survey (2013) The Cost of Having a Baby in the United States: Prepared for Childbirth Connection, Catalyst for Payment Reform and Center for Healthcare Quality and Payment Reform. http://transform.childbirthconnection.org/wp-content/uploads/2013/01/Cost-of-Having-a-Baby1.pdf

[4] Orsi, J.M., Margellos-Anast, H. and Whitman, S. (2010) Black-White Health Disparities in the United States and Chicago: A 15 Year Progress Analysis. American Journal of Public Health, 100, 349-356. http://dx.doi.org/10.2105/AJPH.2009.165407

[5] Moos, M.K. (2006) Prenatal Care: Limitations and Opportunities. Journal of Obstetric, Gynecological and Neonatal Nursing, 35, 278-285. http://dx.doi.org/10.1111/j.1552-6909.2006.00039.x

[6] Sakala, C. and Corry, M.P. (2008). Evidence-Based Maternity Care: What It Is and What It Can Achieve. Milbank Memorial Fund. http://www.milbank.org/reports/0809/MaternityCare/0809MaternityCare.html

[7] Walker, D.S., McCully, L. and Vest, V. (2001) Evidence-Based Prenatal Care: When Less Is More. Journal of Midwifery and Women's Health, 46, 146-151. http://dx.doi.org/10.1016/S1526-9523(01)00120-9

[8] Novick, G. (2004) CenteringPregnancy and the Current State of Prenatal Care. Journal of Midwifery and Women's Health, 49, 405-411. http://dx.doi.org/10.1016/j.jmwh.2004.06.001

[9] Institute of Medicine of the National Academies (2012) Better Care at Lower Cost. http://www.iom.edu/ /media/Files/Report\%20Files/2012/Best-Care/Best\%20Care\%20at\%20Lower\%20Cost_Recs.pdf

[10] Grady, M.A. and Bloom, K.C. (2004) Pregnancy Outcomes of Adolescents Enrolled in a CenteringPregnancy Program. Journal of Midwifery and Women's Health, 49, 412-420. http://dx.doi.org/10.1016/j.jmwh.2004.05.009

[11] Klima, C., Norr, K., Vonderheid, S. and Handler, A. (2009) Introduction of CenteringPregnancy in a Public Health Clinic. Journal of Midwifery and Women's Health, 54, 27-34. http://dx.doi.org/10.1016/j.jmwh.2008.05.008

[12] Trudnak, T.E., Arboleda, E., Kirby, R.S. and Perrin, K. (2013) Outcomes of Latina Women in CenteringPregnancy Group Prenatal Care Compared with Individual Prenatal Care. Journal of Midwifery and Women's Health, 58, 396403. http://dx.doi.org/10.1111/jmwh.12000

[13] Ickovics, J.R., Kershaw, T.S., Westdahl, C., Magriples, U., Massey, Z., Reynolds, H. and Schindler Rising, S. (2007) Group Prenatal Care and Perinatal Outcomes: A Randomized Controlled Trial. Obstetrics and Gynecology, 110, 330339. http://dx.doi.org/10.1097/01.AOG.0000275284.24298.23

[14] Bloom, K.C. (2005) Use of the CenteringPregnancy Program in a School-Based Clinic: A Pilot Study. Clinical Excellence for Nurse Practitioners, 9, 213-218.

[15] Baldwin, K.A. (2006) Comparison of Selected Outcomes of CenteringPregnancy versus Traditional Prenatal Care. Journal of Midwifery and Women's Health, 51, 266-272. http://dx.doi.org/10.1016/j.jmwh.2005.11.011

[16] Ickovics, J.R., Reed, E., Magriples, U., Westdahl, C., Rising, S.S. and Kershaw, T.S. (2011) Effects of Group Prenatal Care on Psychosocial Risk in Pregnancy: Results from a Randomized Controlled Trial. Psychology and Health, 26, 235-250. http://dx.doi.org/10.1080/08870446.2011.531577

[17] Tanner-Smith, E.E., Steinka-Fry, K.T. and Lipsey, M.W. (2013) Effects of CenteringPregnancy Group Prenatal Care 
on Breastfeeding Outcomes. Journal of Midwifery and Women's Health, 58, 389-395. http://dx.doi.org/10.1111/jmwh.12008

[18] Andersson, E., Christensson, K. and Hildingsson, I. (2012) Parents' Experiences and Perceptions of Group-Based Antenatal Care in Four Clinics in Sweden. Midwifery, 28, 502-508. http://dx.doi.org/10.1016/j.midw.2011.07.006

[19] United States Census Bureau (2011) Figure 4: Number in Poverty and Poverty Rate 1959-2011. Income, Poverty and Health Insurance in the United States: 2011-Tables \& Figures. http://www.census.gov/hhes/www/poverty/data/incpovhlth/2011/figure4.pdf

[20] Hoyert, D.L. and Xu, J. (2012) Deaths: Preliminary Data for 2011. National Vital Statistics Report, 61, 1-52. http://www.cdc.gov/nchs/data/nvsr/nvsr61/nvsr61_06.pdf

[21] State of Michigan (2012) Table 3: Number of Infant Deaths, Live Births, and Infant Death Rates by Michigan and Michigan County of Residence, 2011 and 2007-2001 Average. http://www.mdch.state.mi.us/pha/osr/InDxMain/Tab3.asp

[22] United States Census Bureau (2014) Kalamazoo, MI State and County Quick Facts. http://quickfacts.census.gov/qfd/states/26/26077.html

[23] Michigan Department of Community Health (2010) Life Stressors and Smoking Status during Pregnancy. MI PRAMS 2008. MI PRAMS Delivery, 9, 1-4. http://www.michigan.gov/documents/mdch/PRAMS_Newsletter_October_2010_Final_340776_7.pdf

[24] Michigan Department of Community Health (2011) Breastfeeding Initiation and Three Months Exclusive Duration, MI PRAMS 2004-4008. MI PRAMS Delivery, 10, 1-4. http://www.michigan.gov/documents/mdch/PRAMS_Newsletter_January_2011_Final_358253_7.pdf

[25] United States Department of Health and Human Services (2013) 2020 Topics \& Objectives: Maternal, Infant, and Child Health.

[26] Becker, S.O. and Ichino, A. (2002) Estimation of Average Treatment Effects Based on Propensity Scores. The Stata Journal, 2940, 358-377.

[27] Rasmussen, K.M., et al. (2009) Weight Gain during Pregnancy: Reexamining the Guidelines. Institute of Medicine Report Brief. www.iom.edupregnancyweightgain

[28] Centers for Disease Control and Prevention (2002) Women and Smoking: A Report of the Surgeon General (Executive Summary). Morbidity and Mortality Weekly Report, 51, 1-30. http://www.cdc.gov/mmwr/preview/mmwrhtml/rr5112a4.htm

[29] Ogden, C.L., Carroll, M.D., Kit, B.K. and Flegal, K.M. (2012) Prevalence of Obesity in the United States 2009-2010. NCHS Data Brief, No. 82, 1-8. http://www.cdc.gov/nchs/data/databriefs/db82.pdf

[30] Avery, B. and Avery, A. (2012) Healthy Weight Management during Pregnancy: What Advice and Information Is Being Provided? Journal of Human Nutrition and Dietetics, 25, 378-388.

[31] Davis, E. and Olson, C. (2009) Obesity in Pregnancy. Primary Care: Clinics in Office Practice, 36, 341-356. http://dx.doi.org/10.1016/j.pop.2009.01.005

[32] Ramachenderan, J., Bradford, J. and McClean, M. (2008) Maternal Obesity and Pregnancy Complications: A Review. Australian and New Zealand Journal of Obstetrics and Gynaecology, 48, 228-235. http://dx.doi.org/10.1111/j.1479-828X.2008.00860.x

[33] Hoddinott, P., Tappin, D. and Wright, C. (2008) Breast Feeding. British Medical Journal, 336, 881-887. http://dx.doi.org/10.1136/bmj.39521.566296.BE

[34] Connolly, M.E. and Tracewell, R. (2012) Breastfeeding and Obesity. Bariatric Nursing and Surgical Patient Care, 7, 132-135. http://dx.doi.org/10.1089/bar.2012.9967

[35] Centers for Disease Control and Prevention (2010) Breastfeeding Report Card: United States, 2010 (Data File). http://www.cdc.gov/breastfeeding/pdf/Breastfeeding ReportCard2010.pdf

[36] Bergmann, R.L., Bergmann, K.E., Von Weizsäcker, K., Berns, M., Herich, W. and Dudenhausen, J.W. (2014) Breastfeeding Is Natural but Not Always Easy: Intervention for Common Medical Problems of Breastfeeding Mothers-A Review of the Scientific Evidence. Journal of Perinatal Medicine, 42, 9-12. http://dx.doi.org/10.1515/jpm-2013-0095 